DIVISION OF THE HUMANITIES AND SOCIAL SCIENCES

CALIFORNIA INSTITUTE OF TECHNOLOGY

PASADENA, CALIFORNIA 91125

ENVY-FREENESS AND IMPLEMENTATION IN LARGE ECONOMIES

Matthew O. Jackson

California Institute of Technology

Ilan Kremer

Stanford University

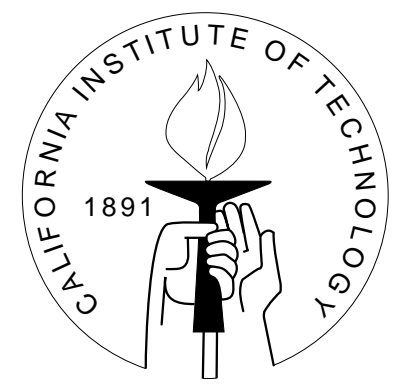

SOCIAL SCIENCE WORKING PAPER 1157

February 2003 


\title{
Envy-Freeness and Implementation in Large Economies
}

\author{
Matthew O. Jackson Ilan Kremer
}

\begin{abstract}
We show that an asymptotic envy-freeness is a necessary condition for a form of robust approximate implementation in large economies.
\end{abstract}

JEL classification numbers: D78, D51, D71 


\title{
Envy-Freeness and Implementation in Large Economies *
}

\author{
Matthew O. Jackson Ilan Kremer
}

\section{Introduction}

The idea that competition leads to efficiency is central to economics. As economies come in many forms and there are many efficient allocations to choose from, it is important have a deep understanding of how far one can push such ideas. The First Welfare Theorem tells us that Walrasian allocations are very well behaved in classical economies with private goods and private ownership of production. However, once we look to other settings and other allocation rules, often the basic insights that underlie the First Welfare Theorem can no longer be applied and the answers are not so clear. Nevertheless, there are many situations where we still might believe that if large numbers of small agents interact then we should be able to implement efficient allocations. So, what exactly is it that defines the situations where this is true? Or more to the point, what conditions identify the settings and social choice functions that we can approximately implement in a large economy?

In this note, we provide a very simple and intuitive answer to the above question. We show that in a wide variety of settings any social choice function that is approximately implementable in large economies (in a strong sense to be made precise) must be asymptotically envy-free. Foley's (1967) property of envy-freeness states that no agent should wish to swap allocations with another agent. The envy-free property is one with a strong normative appeal that has served as the foundation of notions of "fairness." The relationship between this fairness condition and incentives in large economies is embarrassingly simple. It comes in two parts. First, if a social choice function is approximately implemented in a large economy, then it must satisfy an incentive compatibility constraint so that an agent will not pretend to be of another type. Second, approximate implementation requires that in a large enough economy any single agent's announcement will not have much effect on the overall allocation. This means that an agent can obtain almost the same allocation as some other agent by pretending to be of the same type as that other agent. Putting these together we can conclude that an agent does not wish to swap

${ }^{*}$ We are grateful for financial support from the National Science Foundation under grant SES-9986190. We thank Andy Postlewaite for helpful comments on an earlier draft. 
allocations with any other agent in the limit and hence the limiting allocation must be (asymptotically) envy-free.

Beyond envy-freeness being a necessary condition for approximate implementability in large economies, it is in some cases sufficient. We describe a simple variation on a folk-mechanism which provides for approximate implementation of envy-free social choice functions in settings where goods are excludable (thus all private goods and some public goods settings). In the case of non-excludable goods other approaches are necessary. We describe a mechanism that works if certain monotonicity assumptions hold but leave open the question of whether envy-freeness is sufficient for implementation in the most general framework.

\section{Relation to the Literature}

As the question of which allocations are implementable in large economies is fundamental to economics, there are many previous investigations that have shed light on it. Let us mention the most closely related parts of the literature.

To start with, there is a literature that investigates the implementability of the Walrasian correspondence. That literature has looked at small and large economies, complete and incomplete information, full and approximate implementation, and direct and indirect mechanisms. A short summary of some of the main conclusions is as follows. While there are difficulties in implementing Walrasian equilibria in small economies (unless there is complete information), approximating the equilibria in large economies is possible. ${ }^{1}$

Once one moves beyond Walrasian equilibria in a private good exchange economy, the picture is more pessimistic. There is a large literature on mechanisms for the provision of public goods, and there it is also clear (perhaps even clearer) that incentives are a critical issue. However, in public goods settings having large numbers of agents does not necessarily help with incentives. In fact, it can hurt (as shown by Mailath and Postlewaite (1990) and Al-Najjar and Smorodinsky (2000)). The idea there is that in a large economy an agent's announcement has very little chance of influencing the public good decision, and thus agents worry mainly about how their announcement affects their share of the

\footnotetext{
${ }^{1}$ The seminal reference is Hurwicz (1972). A key references on implementation of the Walrasian correspondence under complete information is Hurwicz, Maskin and Postlewaite (1995). Impossibility with small numbers and incomplete information is established in Blume and Easley (1983) and Palfrey and Srivastava (1986). Careful investigation of incentives in large exchange economies began with Roberts and Postlewaite (1976) who showed the gains from manipulation of Walrasian allocations shrink as the economy is replicated. Jackson (1992) and Gul and Postlewaite (1992) showed that in fact the direct Walrasian mechanism is dominant strategy incentive compatible in large economies with a finite set of types. However, the direct mechanism turns out to have problems without the finite type assumption unless some belief restrictions are imposed, as pointed out by Thomson (1979), Otani and Sicilian (1982, 1990) and Jackson and Manelli (1996). If one insists on dominant strategies then even indirect mechanisms will be significantly inefficient in large economies, as shown by Barberà and Jackson (1995). However, as shown by Cordoba and Hammond (1998) and Kovalenkov (2002), some nice approximate implementation results can be recovered if one loosens the balance constraints and works with some clever indirect mechanisms.
} 
cost. Such large economy results on public goods can be obtained as an easy corollary of the envy-free result, as we point out below. Envy-freeness directly implies that all costs must be the same as agents have the same allocation in public goods. ${ }^{2}$

McLean and Postlewaite (2002) is probably the most closely related work in terms of the spirit of trying to uncover the conditions that characterize approximate implementability in large economies. ${ }^{3}$ Their main result is that in pure exchange economies if agents are all "informationally small," then essentially any social choice function can be approximately implemented in the limit. Thus, they are pointing to the fact that no agent should have information that remains essential in the limit, as the defining feature of approximate implementability. The notion of envy-freeness never pops up in their analysis. Let us explain why this is the case. In their setting, uncertainty regards the "characteristics" of goods, and not individuals' preferences over the goods. In particular, in their setting under informational smallness - if you know the information of all but one agent and the economy large enough then you know with arbitrary accuracy what that remaining agent's preferences are. That definition of informational smallness is a strong one, as it precludes agents from having any private information about their own preferences. This is the critical difference, as it is such private information that is the key to implying envy-freeness, and we shall discuss the condition is vacuously satisfied otherwise.

Finally, let us point out that there are two conditions in the way we define social choice functions and implementability that play a role in our results. First, we only consider social choice functions satisfying the well-known "equal treatment of equals" property. This is built directly into our definition of social choice function and is the requirement that if agents differ only in their name and not in any observable or reported characteristics, then they should receive the same allocation. ${ }^{4}$ It is a very minimal anonymity condition. Clearly, if a social choice function always gives everything to agent 1 , just because she is named agent 1 , then that will be implementable and envious. We rule such social choice functions out by fiat. Of course, we do allow the social choice function to depend on what agents announce, and also on other observable characteristics which might be things like the agent's endowment, age, whether they are buyers or sellers, or some pre-existing rights that they have.

The other condition in our definitions that plays a role is the "robustness" of the implementation. This requires that the implementation work even when agents happen to learn extra information about the common state beyond what they know from their type. This condition plays a subtle but important role in our results. In the absence of

\footnotetext{
${ }^{2}$ Note that this is true even under excludability, provided the allocation is efficient.

${ }^{3} \mathrm{Gul}$ and Postlewaite (1992) and Jackson (1992) are perhaps a bit closer to our work in formulation, as they allow for some private aspect to values. However, the motivation of those papers is less in the line of identifying general conditions for implementability. Note also that both of those papers work with variations on Walrasian allocations and so envy-freeness is automatically satisfied.

${ }^{4}$ In our case, this is required from an interim perspective, and identical agents may be receiving random allocations whose ex post realizations might still differ.
} 
such a condition an implementing mechanism can take very strong advantage of small differences in agents' expectations about the state to get them to completely separate themselves. This is the insight that underlies approaches such as that of Crémer and McLean (1988). Such mechanisms, however, are very sensitive to the exact specification of the prior and the agents' information sets. Requiring robustness is a step towards the practical in that it requires a mechanism still perform in situations where agents, either by accident or by their choice, have more information than we presume. This robustness beyond being desirable (and perhaps necessary in practice) is important in deriving envyfreeness. The ability of one agent to pretend to be of another's type is needed to derive envy-freeness and can be prevented if one can rely on non-robust mechanisms where slight differences in information are exploited.

\section{Definitions}

\section{Agents and Economies}

There are countably many agents, who are indexed by $i \in \mathbb{N}$.

We index economies by $n$ indicating the number of agents in the economy, where economy $n$ consists of the first $n$ agents, $\{1, \ldots, n\}$.

\section{Uncertainty, Types, and Information}

Agents have two different sorts of characteristics. Some are openly observable and others are private information. For example, it may be that an agent's age or endowment is observable and that the agent's preferences are private information.

In addition to the potential observed and unobserved heterogeneity of agents, we also wish to have the model allow for some commonality in preferences and payoffs. To this end, a specification of the economy includes a profiles of the agents' public labels, their private types, and a state of nature.

- $L$ is the set of labels, and $\ell_{i} \in L$ denotes agent $i$ 's label.

- $T$ is the set of types, and $t_{i} \in T$ denotes agent $i$ 's type.

- $S$ is the set of states of the world, and the realized state is denoted by $s \in S$.

So, again, $\ell_{i} \in L$ is interpreted as being the publicly observable characteristics of agent $i$ that we call the agent's "label", $t_{i} \in T$ is the agent's private information that we call the agent's "type", and $s \in S$ is a state of nature that can enter into all agents' preferences. 
Depending on the setting and application, what falls into $L$ and what falls into $T$ may vary. For instance, in one application it may be that endowments are observable and preferences are not. In another it may be that neither endowments nor preferences are observable. In yet another application it may be some skill or income that is not observable, while preferences are known.

We make several assumptions on the spaces and the uncertainty.

1. To simplify the exposition we assume that $L, T$, and $S$ are finite. ${ }^{5}$

2. A probability distribution $p$ on $S$ describes the uncertainty on $s$, where $p(s)$ is the probability of state $s \in S$. Types are i.i.d. conditional on the agent's label and the state according to a family of conditional probability measures $p\left(\cdot \mid s, \ell_{i}\right)$ defined on $T$. So, $p\left(t_{i} \mid s, \ell_{i}\right)$ is the probability that an agent is of type $t_{i} \in T$ conditional on the state being $s$ and the agent's label being $\ell_{i}$.

3. Finally, we assume that the proportion of agents of having different labels is welldefined in the limiting economy. That is, for each $l \in L$ there exists $q(l) \in[0,1]$ such that

$$
\frac{\left|\left\{i \leq n \mid \ell_{i}=l\right\}\right|}{n} \rightarrow q(l)
$$

Without loss of generality, let $q(l)>0$ for all $l \in L$.

Note that we are treating labels as given and not uncertain. This is because we assume that they are observable, and so we can think of everything conditional on their realization. A trivial extension would treat them as uncertain without any changes to our results below.

\section{Allocations and Preferences}

$A^{n} \subset \mathbb{R}^{(n+1) k}$ is the set of feasible allocations for economy $n$, where $k$ is some positive integer.

An allocation $a^{n} \in A^{n}$ contains both public and private parts. We write $a^{n}=$ $\left(a_{0}^{n}, a_{1}^{n}, \ldots, a_{n}^{n}\right)$, with the interpretation that $a_{0}^{n}$ is public and may enter any agent's preferences while $a_{i}^{n}$ is something that is private to agent $i$. This is essentially without loss of generality.

Allocations may be quite general, including public goods, private goods, rules, etc.

Let $A_{0}=\cup_{n} A_{0}^{n}, A_{i}=\cup_{n} A_{i}^{n}$, and $A=\cup_{n} A^{n}$.

\footnotetext{
${ }^{5}$ As mentioned in footnote 1 above, distinctions between finite and infinite spaces in such analyses can sometimes be important. In this case it is fairly clear that our results extend to the infinite case, provided some appropriate compactness and continuity conditions are imposed.
} 
It is possible for the set of feasible allocations to depend on agents' labels. However, we require that $A_{i}=A_{j}$ whenever $\ell_{i}=\ell_{j}$.

Agents evaluate allocations according to a function $u: A_{0} \times A_{i} \times S \times L \times T \rightarrow \mathbb{R}$ so that $u\left(a_{0}, a_{i}, s, \ell_{i}, t_{i}\right)$ denotes the utility of allocation $a_{0}, a_{i}$ to an agent of label $\ell_{i}$ and type $t_{i}$ in state $s$.

Having a common $u$ across agents is without loss of generality, as any idiosyncratic aspect of preferences can be built encoded in $t_{i}$ and $\ell_{i}$.

Given an allocation $a$, state $s$ and a type $t_{i}$ of agent $i$, we write $u_{i}\left(a, s, t_{i}\right)$ to denote $u\left(a_{0}, a_{i}, s, \ell_{i}, t_{i}\right)$.

We assume that $u$ is bounded and continuous.

Note that most any economy of interest fits into our setting, including exchange economies, production economies, economies with (excludable or non-excludable) public goods, economies with club goods, commons problems, etc.

\section{Social Choice Functions}

A social choice function on an economy $n$ is a function $f: S \times T^{n} \rightarrow A^{n}$, such that that $\ell_{i}=\ell_{j}$ and $t_{i}=t_{j}$ implies that $a_{i}=a_{j}{ }^{6}$

Note that a social choice function does not take labels as an argument. That is because we treat the labels as known and given, and so the social choice function already can be made to depend on labels.

We build into the definition of a social choice function that it must treat identical agents (in terms of both labels and types) equally. ${ }^{7}$ This still allows agents with different labels, say buyers and sellers, or agents with different endowments, to be treated differently.

\section{Envy-Freeness}

A social choice function $f$ on economy $n$ is $\varepsilon$-envy-free if for every $i$ and $j$ in $\{1, \ldots, n\}$ such that $\ell_{i}=\ell_{j}$

$$
u\left(a_{0}^{n}, a_{i}^{n}, \ell_{i}, t_{i}, s\right) \geq u\left(a_{0}^{n}, a_{j}^{n}, \ell_{i}, t_{i}, s\right)-\varepsilon
$$

\footnotetext{
${ }^{6}$ We may wish to allow for random allocations. This is already admitted under our formulation of the allocation space which lies in a Euclidean space, and $u$ can be an evaluation of expected (or non-expected) utility.

${ }^{7}$ Note that this only needs to hold from the interim perspective. That is, if $a_{i}$ is a random allocation, it simply requires that the distribution over allocations be the same for identical agents, while ex-post they may be treated differently.
} 
for almost every $s$ and $t$, where $a^{n}=f^{n}\left(s, t^{n}\right)$.

Epsilon envy-freeness implies that an agent does not envy the allocation of agents who have the same label by more than some minimal amount. We emphasize that this only applies across agents with the same label.

A sequence of social choice functions $\left\{f^{n}\right\}$ is asymptotically envy-free if for every $\varepsilon>0$ there exists $N$ such that $f^{n}$ is $\varepsilon$ envy-free for all for $n>N$ and $i \leq n$.

\section{Incentive Compatibility and Implementability}

A social choice function $f$ on economy $n$ is incentive compatible if it does not depend on $s$ and satisfies

$$
E\left[u_{i}\left(f\left(t_{-i}, t_{i}\right), t_{i}, s\right) \mid t_{i}\right] \geq E\left[u_{i}\left(f\left(t_{-i}, \widehat{t}_{i}\right), t_{i}, s\right) \mid t_{i}\right],
$$

for all $i, t_{i}$, and $\widehat{t}_{i}$.

The definition of incentive compatibility requires that $f$ depend only on $t$, but not on $s .{ }^{8}$ So, this requires that the social choice function only involve information that can be obtained through the actions or reports of the agents as well as their observable labels but does not require an omniscient social planner who knows the state.

A social choice function $f^{n}$ is robustly incentive compatible if it does not depend on $s$ and satisfies

$$
E\left[u_{i}\left(f^{n}\left(t_{-i}, t_{i}\right), t_{i}, s\right) \mid t_{i}, s\right] \geq E\left[u_{i}\left(f^{n}\left(t_{-i}, \widehat{t}_{i}\right), t_{i}, s\right) \mid t_{i}, s\right]
$$

for all $i, t_{i}, s$, and $\widehat{t}_{i}$.

Robust incentive compatibility requires incentive compatibility even in the case where an agent happens to learn the common part of the state of the world $s$. Note that this is still substantially weaker than ex-post incentive compatibility, as this says nothing about what an agent knows regarding other agents' types - other than the distribution over them - and the social choice function depends only on the agents' types not the state of the world. For instance, in an independent private values world, robust incentive compatibility is exactly equivalent to incentive compatibility. It is very minimal way of ensuring that a mechanism is not sensitive to extra information that agents might learn or might have an incentive to learn. The important aspect of this robust form of incentive compatibility is that it precludes the mechanism from taking advantage of very specific aspects of the correlation structure of uncertainty and hence rules out mechanisms such as those in Crémer and McLean (1988).

\footnotetext{
${ }^{8}$ As already pointed out, $f$ can depend on the labels $\ell_{i}$ as those are given and known.
} 


\section{Implementability}

A sequence of social choice functions $\left\{f^{n}\right\}$ is robustly approximately implementable ${ }^{9}$ if there exists a sequence of robustly incentive compatible $\left\{\hat{f}^{n}\right\}$ so that for each $i$

$$
\left(\widehat{f}_{0}^{n}, \widehat{f}_{i}^{n}\right)-\left(f_{0}^{n}, f_{i}^{n}\right) \rightarrow 0 \text { in probability }
$$

Note that the implemented social choice functions can depend on $s$, while the social choice functions which approximate them can only depend on information obtained from the agents. The idea is that through information obtained from the agents, the state can be estimated and so the implementing social choice functions can come to approximate ones which know the state.

A sequence of social choice functions $\left\{f^{n}\right\}$ is convergent if there exists $f: S \times L \times T \rightarrow$ $A_{0} \times \cup_{i} A_{i}$ (written $\left.f=\left(f_{0}, f_{1}\right)\right)$ such that

$$
\left(f_{0}^{n}, f_{i}^{n}\right)\left[s, t^{n}\right] \rightarrow\left(f_{0}(s), f_{1}\left(s, \ell_{i}, t_{i}\right)\right) \text { in probability }
$$

for each $i$.

A convergent sequence of social choice functions is one that has a well-defined limiting allocation as a function of the state and agent's labels and types.

\section{Envy-Freeness as a Necessary Condition}

We now state the main result.

Theorem 1 A convergent sequence of social choice functions is robustly approximately implementable only if it is asymptotically envy-free.

Proof of Theorem 1: Given is a convergent sequence of social choice functions $\left\{f^{n}\right\}$ with limit $f$ that is robustly approximately implemented by the sequence $\left\{\hat{f}^{n}\right\}$.

We say that the limit $f$ is robustly incentive compatible if for each $s, \ell_{i}, t_{i}$ and $t_{i}^{\prime}$

$$
u\left(f_{0}(s), f_{1}\left(s, \ell_{i}, t_{i}\right), \ell_{i}, s, t_{i}\right) \geq u\left(f_{0}(s), f_{1}\left(s, \ell_{i}, t_{i}^{\prime}\right), \ell_{i}, s, t_{i}\right)
$$

The theorem follows from Lemmas 2, 3 and 4 .

Lemma 2 If a convergent sequence $\left\{f^{n}\right\}$ with limit $f$ is robustly approximately implemented by the sequence $\left\{\hat{f}^{n}\right\}$, then $\left\{\hat{f}^{n}\right\}$ is also convergent with limit $f$.

\footnotetext{
${ }^{9}$ Note that we are using the term implementable in a weak sense in that we are working only with incentive compatibility and not making claims about the full set of equilibria of a mechanism.
} 
Lemma 3 If a convergent sequence $\left\{\hat{f}^{n}\right\}$ is robustly incentive compatible then so is its limit $f$.

Lemma 4 Consider a convergent sequence $\left\{f^{n}\right\}$ of social functions. Its limit $f$ is robustly incentive compatible if and only if the sequence $\left\{f^{n}\right\}$ is asymptotically envy-free.

The proofs of the lemmas appear in the appendix.

A simple application of Theorem 1 is to a public good problem. Consider a society deciding on whether to undertake a costly public project. Agents differ only in the utility they receive from the project and this utility is private information. An immediate corollary of Theorem 1 is that any allocation for large economies which is robustly approximately implementable must have all agents pay the same cost, and so a convergent sequence of social choice functions is robustly approximately implementable only if the cost allocations of agents are identical. This implies that without external financing the public project, if individual rationality constraints are imposed then the project is never built in the limit, which is as shown in Mailath and Postlewaite (1990) and Al-Najjar and Smorodinsky (2000)).

\section{Envy-Freeness as a Sufficient Condition}

Let us briefly describe what we know about the sufficiency of envy-freeness for implementability.

\section{Excludable Goods}

In an excludable goods setting, $A_{0}$ is a singleton. In addition to private goods settings, this admits public goods where agents can be excluded from consuming the public good. In this case envy-freeness is a sufficient condition for robust approximate implementation.

The idea behind this is simple although the details are quite complex. There is a class of "folk" mechanisms for implementation that adapt easily to excludable-good settings. Randomly pick $\sqrt{n}$ agents from the population. Given the excludability, we can ensure that the allocations of these agents are constant across their announcements and so truth is robustly incentive compatible for them. From that sample, with large enough $n$ we learn the state $s$ with arbitrarily high probability. To the remaining agents, we offer a menu based on $f\left(s, \ell_{i}, \cdot\right)$, which given the envy-freeness will be robustly incentive compatible and will approximate the desired social choice functions for large enough $n$ with high probability.

The difficult details regarding making the above heuristic description of a mechanism into a formal statement involve making sure that, for instance, the allocation is at least feasible or perhaps even budget balanced. There are various approaches to dealing with 
balance problems in large economy mechanisms, and since they have been well-studied in the context of exchange economies and extend readily here, we simply refer the interested reader to Cordoba and Hammond (1998) and Kovalenkov (2002) for details.

\section{Non-excludable (Public) Goods}

In situations where we cannot exclude agents from enjoying some goods, the type of mechanism described above is no longer applicable since the sampled agents' incentives are no longer so clear. It is easy to see that some additional conditions will be needed to ensure implementability. For instance, consider a pure public good setting with a binary public good decision (e.g., pass a new law or not), and where there are no transfers available, and agents might like or dislike the project. Any social choice function is necessarily envy-free since the allocation is purely public, and yet there is no way to implement a social choice function that chooses the decision that maximizes total utility (as for instance, agents will exaggerate their like or dislike for the good). In some cases where transfers are possible, one can construct robust implementing mechanisms of envy-free functions. With some nice single- crossing properties and under some specific distributional assumptions, we can charge agents for announcing higher types in proportion to the chance that their announcement turns out to be pivotal in the decision. These differential payments across types disappear in the limit and so we obtain envy-freeness. The question of the full necessary and sufficient conditions for robust approximate im-

plementation in the case where some goods are non-excludable is a difficult one that we leave open.

\section{References}

Al-Najjar, N. and R. Smorodinsky (2000), "Pivotal Players and the Characterization of Influence," Journal of Economic Theory, Vol. 92, pp. 318-342.

Barberà, S. and M.O. Jackson (1995), "Strategy-proof Exchange," Econometrica, Vol. 63, pp. 51-88.

Blume, L. and D. Easley (1983), "Implementation of Rational Expectations Equilibrium with Strategic Behavior," mimeo, Cornell University.

Cordoba, J. and P. Hammond, (1998), "Asymptotically Strategy-proof Walrasian Exchange," Mathematical Social Sciences, Vol. 36, pp 185-212.

Foley, D. (1967), "Resource Allocation and the Public Sector," Yale Economic Essays, Vol. 7, pp. 45-98. 
Gul, F. and A. Postlewaite (1992), "Asymptotic Efficiency in Large Exchange Economies with Asymmetric Information," Econometrica, Vol. 60, pp. 1273-1292.

Hurwicz, L. (1972), "On Informationally Decentralized Systems." In C.B. McGuire and R. Radner, Decision and Organization, North Holland, Amsterdam.

Hurwicz, L., E. Maskin, and A. Postlewaite (1995), "Feasible Nash Implementation of Social Choice Correspondences when the Designer does not Know Endowments or Production Sets." In J. Ledyard, The Economics of Information Decentralization: Complexity, Efficiency, and Stability, Kluwer, Amsterdam.

Jackson, M.O. (1992), "Incentive Compatibility and Competitive Allocations," Economics Letters, Vol. 40, pp. 299-302.

Jackson, M.O. and A. Manelli (1996), "Approximate Competitive Equilibria in Large Economies," Journal of Economic Theory, Vol. 77, pp. 354-376.

Kovalenkov, A(2002) "On a Folk Strategy-Proof Approximately Walrasian Mechanism," Journal of Economic Theory, 103, pp 475-487.

Mailath, G. and A. Postlewaite (1990), "Asymmetric Information Bargaining Problems with Many Agents," Review of Economic Studies, Vol. 57, pp. 351-367.

McLean, R. and A. Postlewaite (2002), "Informational Size and Incentive Compatibility," Econometrica, Vol. 70, pp. 2421-2454.

Otani, Y. and J. Sicilian (1982), "Equilibrium Allocations of Walrasian Preference Games," Journal of Economic Theory, Vol. 27, pp. 47-68.

Otani, Y. and J. Sicilian (1990), "Limit Properties of Equilibrium Allocations of Walrasian Strategic Games," Journal of Economic Theory, Vol. 51, pp. 295-312.

Palfrey, T. and S. Srivastava (1987), "On Bayesian Implementable Allocations," The Review of Economic Studies, Vol. 54, pp. 193-208.

Roberts, D.J. and A. Postlewaite,(1976) " The Incentives for Price Taking Behavior in Large Exchange Economies," Econometrica,Vol. 44,115-127.

Thomson, W.,(1979)"Equilibrium Allocations of Walras and Lindahl Manipulation Games,"DP 111, Center for Economic Research, University of Minnesota. 


\section{Appendix}

Proof of Lemma 2: This follows from the finiteness of the state and type spaces, which give us uniform convergence of both sequences.

Proof of Lemma 3: Consider a convergent sequence $\left\{\hat{f}^{n}\right\}$ that is robustly incentive compatible. It follows that

$$
E\left[u\left(\hat{f}_{0}^{n}\left(t_{-i}, t_{i}\right), \widehat{f}_{i}^{n}\left(t_{-i}, t_{i}\right), t_{i}, s\right) \mid s, t_{i}\right] \geq E\left[u\left(\widehat{f}_{0}^{n}\left(t_{-i}, t_{i}^{\prime}\right), \widehat{f}_{i}^{n}\left(t_{-i}, t_{i}^{\prime}\right), t_{i}, s\right) \mid s, t_{i}\right]
$$

Observe that not only $\hat{f}_{0}^{n}\left(t_{-i}, t_{i}\right)$ but also $\hat{f}_{0}^{n}\left(t_{-i}, t_{i}^{\prime}\right)$ converges to $f_{0}(s)$ in probability. That is, in the limit if an agent misrepresents his type he does not change the public part of the allocation. To see this we consider $\hat{f}_{0}^{n}\left(t_{-i}, t^{*}\right)$ for some fixed type $t^{*}$. Conditional on the state being $s$ there is a positive probability that $t_{i}=t^{*}$. Hence, the fact that $\widehat{f}_{0}^{n}\left(t_{-i}, t_{i}\right)$ converges to $f_{0}(s)$ in probability implies that also $\hat{f}_{0}^{n}\left(t_{-i}, t^{*}\right)$ converges to $f_{0}(s)$ in probability.

Since for any $s$ and $\ell_{i}, t_{i}$,

$$
\left(\widehat{f}_{0}^{n}, \widehat{f}_{i}^{n}\right)\left[s, t_{-i}^{n}, t_{i}\right] \rightarrow f_{0}(s), f_{1}\left(s, \ell_{i}, t_{i}\right) \text { in probability, }
$$

it follows that that for any $t_{i}^{\prime}$

$$
\left(\widehat{f}_{0}^{n}, \widehat{f}_{i}^{n}\right)\left[s, t_{-i}^{n}, t_{i}^{\prime}\right] \rightarrow f_{0}(s), f_{1}\left(s, \ell_{i}, t_{i}^{\prime}\right) \text { in probability }
$$

Since $u$ is continuous and bounded, given the robust incentive compatibility of the sequence, the claim then follows from (1).

Proof of Lemma 4: First, suppose that $\left\{f^{n}\right\}$ is asymptotically envy-free but that $f$ is not robustly incentive compatible so that there is some $s, \ell_{i}, t_{i}$ and $t_{i}^{\prime}$, and $\varepsilon^{*}$ such that

$$
u\left(f_{0}(s), f_{1}\left(s, \ell_{i}, t_{i}\right), \ell_{i}, s, t_{i}\right)<u\left(f_{0}(s), f\left(s, \ell_{i}, t_{i}^{\prime}\right), \ell_{i}, s, t_{i}\right)-\varepsilon^{*} .
$$

Since $p\left(t_{i}^{\prime} \mid s, \ell_{i}\right)>0$ and since $q\left(\ell_{i}\right)>0$ we can almost surely find a $j$ so that $\ell_{j}=\ell_{i}$ and $t_{j}=t_{i}^{\prime}$. This leads to a contradiction of asymptotic envy-freeness as $f_{j}^{n}\left(s, t_{-j}, t_{j}\right) \rightarrow$ $f_{1}\left(s, \ell_{i}, t_{i}^{\prime}\right)$ (in probability, independently of $\left.t_{i}\right)$ and $f_{i}^{n}\left(s, \ell_{i}, t_{-i}, t_{i}\right) \rightarrow f_{1}\left(s, \ell_{i}, t_{i}\right)$ (in probability, independently of $t_{j}$ ), and so $j$ will envy $i$ by a fixed amount, even for large $n$.

Next consider the converse. Suppose that $f$ is robustly incentive compatible, but $\left\{f^{n}\right\}$ is not asymptotically envy-free. Since $L, S$ and $T$ are finite, we can find $s, \ell_{i}, t_{i}$ and an $\varepsilon^{*}$ so that for arbitrary large $n$, some agent $i$ who has label $\ell_{i}$ and type $t_{i}$ envies another agent $j$ of type $t_{j}$ where $\ell_{j}=\ell_{i}$ by more than $\varepsilon^{*}$ :

$$
u\left(f_{0}^{n}(s, t), f_{i}^{n}(s, t), \ell_{i}, t_{i}, s\right)<u\left(f_{0}^{n}(s, t), f_{j}^{n}(s, t), \ell_{i}, t_{i}, s\right)-\varepsilon^{*}
$$

$f^{n}$ being convergent to $f$ implies that:

$$
\left\{f_{0}^{n}\left(s, t_{-i}, t_{j}\right), f_{i}^{n}\left(s, t_{-i}, t_{j}\right)\right\} \rightarrow\left(f_{0}(s), f_{1}\left(s, \ell_{i}, t_{j}\right)\right) \text { in probability. }
$$

Hence, a contradiction of robust incentive compatibility of the limit $f$ follows, noting that $u$ is bounded and continuous. 